\title{
Botany
}

\section{HIDE AND SEEK: MOLECULAR BARCODING CLARIFIES THE DISTRIBUTION OF TWO CRYPTIC DUCKWEED SPECIES ACROSS ALBERTA}

\begin{tabular}{|r|l|}
\hline Journal: & Botany \\
\hline Manuscript ID & cjb-2021-0058.R1 \\
\hline Manuscript Type: & Note \\
\hline Author: & $17-J u n-2021$ \\
\hline Complete List of Authors: & $\begin{array}{l}\text { Senevirathna, Kanishka M.; University of Lethbridge, Department of } \\
\text { Biological Sciences } \\
\text { Crisfield, Varina E.; Royal Alberta Museum, Alberta Biodiversity } \\
\text { Monitoring Institute, ; University of Sherbrooke } \\
\text { Burg, Theresa M.; University of Lethbridge, Department of Biological } \\
\text { Sciences } \\
\text { Laird, Robert A.; University of Lethbridge, Department of Biological } \\
\text { Sciences }\end{array}$ \\
\hline Keyword: & $\begin{array}{l}\text { Biogeography, DNA barcoding, Lemna minor, Lemna turionifera, } \\
\text { Molecular markers }\end{array}$ \\
\hline $\begin{array}{r}\text { Is the invited manuscript for } \\
\text { consideration in a Special } \\
\text { Issue? : }\end{array}$ & \begin{tabular}{l} 
Not applicable (regular submission) \\
\hline
\end{tabular} \\
\hline
\end{tabular}

\section{SCHOLARONE Manuscripts}




\title{
HIDE AND SEEK: MOLECULAR BARCODING CLARIFIES THE DISTRIBUTION OF TWO CRYPTIC DUCKWEED SPECIES ACROSS ALBERTA
}

\author{
Kanishka M. Senevirathna*,1,4, Varina E. Crisfield ${ }^{2,3}$, Theresa M. Burg ${ }^{1}$, and Robert A. Laird ${ }^{1}$ \\ ${ }^{1}$ Department of Biological Sciences, University of Lethbridge, Lethbridge, AB T1K 3M4, \\ Canada \\ ${ }^{2}$ Alberta Biodiversity Monitoring Institute, Royal Alberta Museum, 9810 103A Avenue \\ Edmonton, Alberta, T5J 0G2 \\ ${ }^{3}$ University of Sherbrooke, 2500, boulevard de l'Université, Sherbrooke (Québec) J1K 2R1 \\ ${ }^{4}$ Department of Agricultural, Food, and Nutritional Science, 4-10 Agriculture/Forestry Centre, \\ University of Alberta, Edmonton, AB, T6G 2P5 \\ *Corresponding author: kanishkamadushanka12191@gmail.com
}




\begin{abstract}
Regional and global biodiversity may be underestimated due to the presence of cryptic species: species that are morphologically similar, but genetically distinct. Here we focus on two cryptic duckweed species, Lemna minor and L. turionifera, which have overlapping geographic ranges and are easily mistaken for one another. We developed species-specific primers based on DNA barcoding sequences to facilitate the rapid identification of these two monomorphic duckweeds, allowing us to investigate their presence and distribution in Alberta, Canada. While current reports indicate the presence of $L$. turionifera (and the morphologically distinct L. trisulca) in Alberta, our data indicate that L. minor is also present, predominantly in the southern part of the province. Thus, this paper (1) contributes to the accuracy and completeness of a regional flora, and (2) provides useful and flexible tools for the rapid molecular identification of cryptic Lemna species, species of wide interest in such diverse fields as biotechnology, toxicology, bioremediation, and ecology.
\end{abstract}

Keywords: Biogeography, DNA barcoding, Lemna minor, Lemna turionifera, Molecular markers 


\section{Introduction}

Regional and global biodiversity may be underestimated because of the presence of cryptic species, i.e., those that are morphologically identical, but genetically distinct (Chenuil et al., 2019). Molecular tools, specifically DNA barcoding, have improved the ability to identify cryptic species, allowing more detailed assessments of biodiversity than via morphological analyses alone (Hollingsworth et al., 2011).

A prominent plant group that contains cryptic species is the duckweeds (Subfamily Lemnoideae), the smallest aquatic monocots. Possibly contributing to a propensity for crypsis, duckweeds have simple morphology, being composed of a single frond (the lens-shaped body of the plant, comprising the leaf and stem) and zero to several roots, depending on the species (Landolt, 1975, Lemon and Posluszny, 2000). These fast-growing, free-floating plants are widespread throughout freshwater bodies (Keddy, 1976, Landolt, 1986) with 36 recognized species in five genera (Spirodela Schleid, Landoltia Les \& Crawford, Lemna L., Wolffiella Hegelm, and Wolffia Horkel ex Schleid) (Bog et al., 2020).

The genus Lemna is found on every continent except Antarctica and is most diverse in North America and southeast Asia (Landolt, 1975). Recently, Lemna species have attracted attention due to their ecological and economic significance (Xu et al., 2012, Tang et al., 2014, Barks et al., 2018). Lemna plays an important ecological role as a food source for various waterfowl and fish species (Goopy and Murray, 2003) and is economically important in aspects of environmental biotechnology, such as feedstock and biofuel production (Cheng and Stomp, 2009, Xu et al., 2012, Van der Spiegel et al., 2013). Further, duckweeds are used for biomonitoring, phytoremediation, phytohormone biosynthesis and photosynthesis studies (Bog et al., 2010, Ziegler et al., 2015), and as model organisms for comparative studies (Böttcher and 
Schroll, 2007), ecotoxicological studies (Hulsen et al., 2002, Aliferis et al., 2009), and studies of pathogenesis (Zhang et al., 2010). Lemna species are also used in quantitative analyses (e.g., demographic studies) due to their small stature, simple morphology, rapid and mainly clonal reproduction, and widespread distribution (Jansen et al., 1996, Laird and Barks, 2018). Their simple morphology has led to problems with accurately distinguishing among many species, due to their similar appearance. As such, additional methods need to be developed to quickly and accurately identify Lemna species to facilitate obtaining basic information such as geographic range and taxonomic certainty (Bog et al., 2020a; Marconi et al., 2019).

Lemna species are widespread across the Canadian province of Alberta (VASCAN, 2019, ABMI, 2020, Flora of North America, 2020). They are an important food source for many of Alberta's aquatic animal species (e.g., beavers). To date, the most basic biodiversity question "How many Lemna species are there in Alberta?" has not yet been answered conclusively (Barks et al., 2018). According to the Alberta Biodiversity Monitoring Institute (ABMI, 2020) and other data sources (VASCAN, 2019, Flora of North America, 2020), Alberta hosts two morphologically distinct Lemna species: L. trisulca and L. turionifera (ABMI, 2020). DNA barcoding data suggest the presence of a third Lemna species, L. minor, at a single site in southeast Alberta (Crow Indian Lake, Skiff, AB; $49.37^{\circ} \mathrm{N}, 111.80^{\circ} \mathrm{W}$ ) (Barks et al., 2018). Lemna turionifera and L. minor have extremely similar morphologies and are cryptic species (Landolt, 1986, Wang et al., 2010). Morphological studies indicate that $L$. turionifera and $L$. minor can be differentiated by the red pigmentation on the abaxial surface and papules along the midline of the adaxial surface of L. turionifera (Dudley, 1987). In addition, L. turionifera produces starchy overwintering buds called turions (Flora of North America, 2020). However, Dudley (1987) reported that turions are also present in L. minor, and the other traits are 
insufficiently diagnostic given variation among strains; therefore, it is unknown whether $L$. minor is rare and/or geographically restricted within Alberta, or simply 'camouflaged' by its resemblance to L. turionifera.

In this study, we developed molecular tools to differentiate between strains of Lemna and to assess the biogeographical distribution of the cryptic species L. turionifera and L. minor in Alberta. We determined that L. minor is present at several sites in Alberta and appears to be particularly common in the south. More broadly, our development of species-specific primers allows for rapid screening of large numbers of samples without resorting to expensive and timeconsuming DNA sequencing.

\section{Materials and Methods}

\section{Sampling of Lemna}

To determine the species distribution and assess population genetics of $L$. minor and $L$. turionifera, we sampled 126 water bodies throughout Alberta, collecting 234 Lemna samples (Supplemental Table S1). Sampling was done in collaboration with the ABMI and the Royal Alberta Museum. ABMI provided 57 samples of Lemna collected between 2016 and 2018. Full ABMI sampling protocols can be found in ABMI (2016); however, salient details are as follows. ABMI wetland sites include natural or artificial wetlands at least 1 ha in size and $0.5 \mathrm{~m}$ deep in July. Plant surveys were conducted along $5 \times 2 \mathrm{~m}$ transects located in each of four wetland zones (open water, emergent, graminoid, and wooded). The number of transects varied according to the number of zones present at each site. Lemna was sampled from a single location in each wetland, in the transect in which it was first encountered. Samples consisted of numerous fronds, which 
were dried, pressed, and sent to the Royal Alberta Museum for verification and preservation. All samples were transferred to archival storage media upon receipt.

Another 77 Lemna samples were collected at 27 sites in 2013 and initially used in Barks et al. (2018). In the summer of 2019, we collected another 100 samples from 42 additional sites (Alberta Tourism, Parks and Recreation, Parks Division Permit No: 19-522). These sites included ponds, slough, lakes, and potholes throughout Alberta. Approximately one to three clumps of Lemna were collected per site and either stored in ethanol or brought back to the lab for cultures and then stored in ethanol.

We also used seven reference strains used in Barks et al. (2018): three from the Rutgers Duckweed Stock Cooperative (RDSC; L. minor RDSC 7123, S. polyrhiza RDSC 8790, and L. minuta RDSC 6752) and four from the Canadian Phycological Culture Centre (CPCC; L. gibba CPCC 310, L. minor CPCC 490 and CPCC 492, and L. trisulca CPCC 399) (Supplemental Table S1).

\section{Development of atpF-atpH and psbK-psbI species-specific primers}

We used atpF-atpH and $p s b K-p s b I$ sequences from Wang et al. (2010), Tang et al. (2014) and Barks et al. (2018) to create species-specific primers for five duckweed species $(L$. turionifera, L. minor, S. polyrhiza, L. minuta, and L. gibba) with varying degrees of morphological similarity, all of which occur in North America. Although L. trisulca is present throughout Alberta, we did not design any primers for this species because it is morphologically distinct, even when examining dried or damaged specimens. We downloaded 33 sequences from National Center for Biotechnology Information (NCBI) (Wang et al., 2010, Tang et al., 2014, Barks et al., 2018), 17 for atpF-atpH (MG000358, MG000413, MG000505, MG000371-373, MG00416, MG000397, MG000405, MG000406, GU454232, GU454233, GU454206-208, 
KP017659, and KP017648) and 16 for psbK-psbI (MG000432, MG000487, MG000479, MG000480, MG000471, MG000490, MG000445-447, GU454338, GU454328-330, and GU454300-302) from the five duckweed species. We aligned sequences using MEGA 6.0 (Tamura et al., 2013) and identified species-specific target sites (Supplemental Figures S1-S4, Supplemental Table S1). We designed eight species-specific Lemna primers using Primer3 Input 0.4.0 (https://bioinfo.ut.ee/primer3-0.4.0/), allowing us to screen large numbers of samples without sequencing every individual.

\section{Screening Lemna species using atpF-atpH and psbK-psbI species-specific primers}

First, we tested the species-specific primers against the reference strains to demonstrate that they amplified only the target species (Figure 1). For screening and identification, we extracted total DNA from one frond for each sample using a Geneaid Genomic DNA Mini Kit (Plant: GP100; FroggaBio Inc, Concord, ON, Canada). To increase the accuracy of the screening process, we randomly selected and extracted DNA from five individuals for each waterbody. We tested each primer on our five target duckweed species (L. turionifera, L. minor, as well as $L$. gibba, L. minuta, and S. polyrhiza). We also tested the primers against the morphologically distinct $L$. trisulca that is also present in Alberta. We performed DNA amplification using an Eppendorf master cycler using the eight species-specific primers. PCRs were performed in $10 \mu \mathrm{l}$ reactions, with $1 \times$ Truin buffer, $0.2 \mathrm{mM} \mathrm{dNTP}, 0.2 \mathrm{mM}$ of each forward and reverse primer and 1 Unit tru taq (Barks et al., 2018). PCR products were run on an agarose gel to verify the presence of amplified DNA fragments.

All samples were initially screened with an $L$. turionifera specific $p s b K-p s b I$ primer as $L$. turionifera is the most common species in the study area (Barks et al., 2018). Any sample that did not amplify with $L$. turionifera primers was screened with $L$. minor specific atpF-atpH and 
psbK-psbI primers (Table 1), as L. minor is the other morphologically similar species believed to be present in Alberta. If a sample did not amplify with either of those primers, it was reamplified with all eight species-specific primers to determine if the initial PCR failed or if it was another species.

\section{Results}

\section{Development of atpF-atpH and psbK-psbI species-specific primers}

Nucleotide sequence differences in both atpF-atpH and $p s b K$ - $p s b I$ allowed us to develop eight species-specific primers for five morphologically similar North American duckweed species: $L$. turionifera, L. minor, L. gibba, L. minuta, and S. polyrhiza (Supplemental Figures S1 and S2) each with unique banding profiles (Figure 1). We subsequently checked the primer sequences against published sequences for other Lemna species and found that in three instances, the primers could amplify multiple species. Specifically, the two L. minor primers (LemnaMrpsb97_F and LemnaMratp152_R) will also amplify L. japonica, the L. minuta primers (LemnaMapsb178_F and LemnaMaatp236_F) will amplify L. yungensis (psbK-psbI only) and L. valdiviana, and the two L. gibba primers (LemnaGpsb241_F and LemnaGatp394_F) will also amplify L. disperma. For the last two species pairs, sequences are distinct enough for both loci that primers could easily be developed to separate them using the same techniques described here. Additionally, when factoring in the geographic distributions of the species, of the above species groups, only L. minuta and L. valdiviana have overlapping ranges (Landolt, 1986).

\section{Screening Lemna spp. using atpF-atpH and psbK-psbI species-specific primers}

Of the 126 sites, $91.3 \%$ (115) contained L. turionifera only, 3.2\% (4) contained L. minor only, and 5.5\% (7) contained both L. turionifera and L. minor. All but one of the sites containing $L$. 
minor were in the southern part of the province (the exception being found at $57.24^{\circ} \mathrm{N}, 115.91^{\circ}$ W) (Figure 2).

\section{Discussion}

Due to the morphological similarity and overlapping ranges of L. turionifera and L. minor, the identities of Lemna species in Alberta has been an open question. We successfully used DNA barcoding to differentiate five morphologically similar Lemna species. These primers allowed us to screen large numbers of samples across Alberta and outline their distributions. Our data show L. minor is present in multiple wetlands in southern Alberta (see Supplemental Table S1) and a single site in the far north of the province $\left(57.24^{\circ} \mathrm{N}, 115.91^{\circ} \mathrm{W}\right)$. All the southern L. minor sites in Alberta are in the South Saskatchewan River Basin, which includes the Red Deer, Bow, and Oldman Rivers. The northern outlier may be a result of long-distance dispersal, potentially by migrating waterbirds (Coughlan et al., 2017a, Coughlan et al., 2017b). Given its northerly location, this occurrence may represent an ephemeral population that is unlikely to persist; however, this cannot be confirmed without repeat sampling.

Our finding of the overlapping distribution of L. minor and L. turionifera in Alberta raises several questions. For example, why do some sites have only one of the two Lemna species? And why do other sites contain both Lemna species? In this study we report coexistence of $L$. minor and L. turionifera at seven of the 126 water bodies. It is possible that many candidate sites have both Lemna species, but that one species is more abundant, such that we only detected one despite screening five individuals for each water body. Alternatively, perhaps differences in water quality restrict the distribution of one species, such that overlap is rare, or one species is better adapted, conferring a fitness advantage. Environmental traits related to 
water chemistry (e.g., pH, conductivity, nutrient concentrations) and interspecific competition between species of duckweed can affect Lemna species' distributions (Landolt, 1975, Armitage and Jones, 2020). For example, Landolt (1975) reported the range of $\mathrm{pH}$ for L. minor is 5.0-7.5 and 3.5-8.2 for L. turionifera, and the range for water conductivity for L. minor is 70-700 $\mathrm{mS} / \mathrm{cm}$, compared to $185-1,300 \mathrm{mS} / \mathrm{cm}$ for L. turionifera.

At large geographic scales, the distribution of Lemna species is limited by climatic boundaries and precipitation (Landolt, 1975). Lemna turionifera inhabits the majority of the continental areas of eastern Asia and North America characterized by lower temperatures and low amounts of precipitation. In contrast, L. minor grows abundantly in moister climates, being rare or absent in more arid regions (Landolt, 1975). Our results suggest that the geographic distribution of the two Lemna species is quite different in Alberta, with L. minor primarily restricted to the drier southern regions of the province. Climate data collected over past three decades (Daly et al., 2010) show the average annual precipitation in Alberta is generally higher in the northwest and lowest in the southeast. The pattern is reversed for average annual temperatures. Thus, the overall geographic pattern of Lemna in Alberta runs counter to that expected based on Landolt (1975).

An outstanding issue with our approach is that our primers were designed based only on species relevant to the study area, and may not uniquely discriminate these species among all duckweeds worldwide. While primers for three of the species could amplify in one other species (see results), designing new primers and using additional information such as location would allow us to differentiate them. Lemna minuta and L. valdiviana have ranges that overlap, however, the two are genetically distinct (Landolt, 1986). Lemna gibba and L. disperma have different sequences and are found in different geographic areas (Landolt, 1986). For L. minor 
and L. japonica, published GenBank sequences are too similar for species-specific primers, though they inhabit different areas. Anthropogenic activities such as species introductions and climate change could alter species' distributions (Bykova et al., 2012). In light of this, we encourage the development of species-specific primers for all species of duckweed using same methodology described in this paper.

The DNA barcoding method used in this study allows for species identification and largescale screening of L. minor and L. turionifera. The same barcoding techniques in this study can be used to reassess the taxonomic status of herbarium specimens of Lemna. These assessments will be important to determine whether and how these cryptic species affect regional species richness, as well as range size and distributional limits.

\section{Acknowledgements}

We thank the Alberta Biodiversity Monitoring Institute, the Royal Alberta Museum, Donna Cherniawsky, and Patrick Barks for providing samples. We thank Suzanne Chmilar, Ian Gazeley and Brendan Graham for assistance in the lab and field. This research was supported by an Alberta Conservation Association Research Grant (grant no. 015-00-90-281 [RAL and TMB]) and two Natural Sciences and Engineering Research Council of Canada Discovery Grants (grant no. RGPIN-2015-05486 [RAL] and 2019-05068 [TMB]). 


\section{References}

Alberta Biodiversity Monitoring Institute (ABMI), 2016, Wetland Field Data Collection Protocols (Abridged Version) 2016-05-26. Alberta Biodiversity Monitoring Institute, Alberta, Canada. Report available at: abmi.ca

ABMI. 2020. Alberta Biodiversity Monitoring Institute. Vascular plants [Online]. Available: https://abmi.ca/home/data-analytics/biobrowser-home/species-list?groupId=2 [Accessed October $\left.26^{\text {th }} 2020\right]$.

Aliferis, K. A., Materzok, S., Paziotou, G. N. and Chrysayi-Tokousbalides, M. 2009. Lemna minor L. as a model organism for ecotoxicological studies performing $1 \mathrm{H}$ NMR fingerprinting. Chemosphere, 76, 967-973.

Armitage, D. W. and Jones, S. E. 2020. Coexistence barriers confine the poleward range of a globally distributed plant. Ecol. Lett., 23, 1838-1848.

Barks, P. M., Dempsey, Z. W., Burg, T. M. and Laird, R. A. 2018. Among-strain consistency in the pace and shape of senescence in duckweed. J. Ecol., 106, 2132-2145.

Bog, M., Baumbach, H., Schween, U., Hellwig, F., Landolt, E. and Appenroth, K. J. 2010. Genetic structure of the genus Lemna L.(Lemnaceae) as revealed by amplified fragment length polymorphism. Planta, 232, 609-619.

Bog, M., Himmelbach, A., Brandt, R., Wagner, F., and Appenroth, K. J. 2020. "Genotyping-BySequencing for species delimitation in Lemna section Uninerves Hegelm. (Lemnaceae), In The Duckweed Genomes. Edited by X. H. Cao, P. Fourounjian, and W. Wang. Springer, Berlin. pp. 115-123. doi: 10.1007/978-3-030-11045-1_11

Böttcher, T. and Schroll, R. 2007. The fate of isoproturon in a freshwater microcosm with Lemna minor as a model organism. Chemosphere, 66, 684-689.

Bykova, O., Chuine, I., Morin, X. and Higgins, S. I. 2012. Temperature dependence of the reproduction niche and its relevance for plant species distributions. J. Biogeogr., 39, 2191-2200.

Cheng, J. J. and Stomp, A. M. 2009. Growing duckweed to recover nutrients from wastewaters and for production of fuel ethanol and animal feed. CLEAN-Soil, Air, Water, 37, 17-26.

Chenuil, A., Cahill, A.E., Délémontey, N., Du Salliant du Luc, E., and Fanton, H. 2019. Problems and Questions Posed by Cryptic Species. A Framework to Guide Future Studies. In From Assessing to Conserving Biodiversity. History, Philosophy and Theory of the Life Sciences, vol 24. Edited by E. Casetta, J. Marques da Silva, and D. Vecchi. Springer, Cham. doi: 10.1007/978-3-030-10991-2_4

Coughlan, N. E., Kelly, T. C., Davenport, J. and Jansen, M. A. 2017a. Up, up and away: Bird-mediated ectozoochorous dispersal between aquatic environments. Freshwat. Biol., 62, 631-648. 
Coughlan, N. E., Kelly, T. C. and Jansen, M. A. 2017b. "Step by step": high frequency shortdistance epizoochorous dispersal of aquatic macrophytes. Biol. Invasions, 19, 625-634.

Daly, C., Conklin, D. R. and Unsworth, M. H. 2010. Local atmospheric decoupling in complex topography alters climate change impacts. Int. J. Climatol., 30, 1857-1864.

Dudley, J. L. 1987. Turion formation in strains of Lemna minor (6591) and Lemna turionifera (6573, A). Aquat. Bot., 27, 207-215.

Flora of North America. 2020. Lemna L. Web documents [Online]. Available:

http://www.efloras.org/florataxon.aspx?flora_id=1\&taxon_id=117936\#KEY-1-7, http://www.efloras.org/object_page.aspx?object_id=11231\&flora_id=1, http://www.efloras.org/object_page.aspx?object_id=11248\&flora_id=1, http://www.efloras.org/object_page.aspx?object_id=11244\&flora_id=1 . [Accessed October $26^{\text {th }}$ 2020].

Goopy, J. P. and Murray, P. 2003. A review on the role of duckweed in nutrient reclamation and as a source of animal feed. Asian-australas. J. Anim. Sci., 16, 297-305.

Hollingsworth, P. M., Graham, S. W. and Little, D. P. 2011. Choosing and using a plant DNA barcode. PloS One, 6, e19254.

Hulsen, K., Minne, V., Lootens, P., Vandecasteele, P. and Höfte, M. 2002. A chlorophyll a fluorescence-based Lemna minor bioassay to monitor microbial degradation of nanomolar to micromolar concentrations of linuron. Environ. Microbiol., 4, 327-337.

Jansen, M. A., Gaba, V., Greenberg, B. M., Mattoo, A. K. and Edelman, M. 1996. Low threshold levels of ultraviolet- $\mathrm{B}$ in a background of photosynthetically active radiation trigger rapid degradation of the D2 protein of photosystem-II. The Plant Journal, 9, 693-699.

Keddy, P. A. 1976. Lakes as islands: the distributional ecology of two aquatic plants, Lemna minor L. and L. trisulca L. J. Ecol., 57, 353-359.

Laird, R. A. and Barks, P. M. 2018. Skimming the surface: duckweed as a model system in ecology and evolution. Am. J. Bot., 105, 1962-1966.

Landolt, E. 1975. Morphological differentiation and geographical distribution of the Lemna gibba-Lemna minor group. Aquat. Bot., 1, 345-363.

Landolt, E. 1986. Biosystematic investigations in the family of duckweeds (Lemnaceae), Veröffentlichungen des Geobotanischen Institutes der ETH, Siftung, Rübel, Zürich, Switzerland.

Lemon, G. D. and Posluszny, U. 2000. Comparative shoot development and evolution in the Lemnaceae. Int. J. Plant Sci., 161, 733-748.

Tamura, K., Stecher, G., Peterson, D., Filipski, A. and Kumar, S. 2013. MEGA6: molecular evolutionary genetics analysis version 6.0. Mol. Biol. Evol., 30, 2725-2729. 
Tang, J., Zhang, F., Cui, W. and Ma, J. 2014. Genetic structure of duckweed population of Spirodela, Landoltia and Lemna from Lake Tai, China. Planta, 239, 1299-1307.

Van der Spiegel, M., Noordam, M. and Van der Fels-Klerx, H. 2013. Safety of novel protein sources (insects, microalgae, seaweed, duckweed, and rapeseed) and legislative aspects for their application in food and feed production. Compr. Rev. Food Sci. Food Saf., 12, 662-678.

VASCAN. 2019. Database of vascular plants of Canada (VASCAN). [Online]. Available: http://data.canadensys.net/vascan/taxon/6489?lang=en, http://data.canadensys.net/vascan/taxon/6491?lang=en, http://data.canadensys.net/vascan/taxon/6492?lang=en [Accessed October $26^{\text {th }} 2020$ ].

Wang, W., Wu, Y., Yan, Y., Ermakova, M., Kerstetter, R. and Messing, J. 2010. DNA barcoding of the Lemnaceae, a family of aquatic monocots. BMC Plant Biol., 10, 205.

Xu, J., Zhao, H., Stomp, A.-M. and Cheng, J. J. 2012. The production of duckweed as a source of biofuels. Biofuels, 3, 589-601.

Zhang, Y., Hu, Y., Yang, B., Ma, F., Lu, P., Li, L., Wan, C., Rayner, S. and Chen, S. 2010. Duckweed (Lemna minor) as a model plant system for the study of human microbial pathogenesis. PLoS One, 5, e13527.

Ziegler, P., Adelmann, K., Zimmer, S., Schmidt, C. and Appenroth, K. J. 2015. Relative in vitro growth rates of duckweeds (Lemnaceae)-the most rapidly growing higher plants. Plant Biol., 17, 33-41. 


\section{Tables}

Table 1. Species-specific* Lemna PCR primers and length of PCR product (bp) designed for this study. Universal reverse primers were used for each locus: $p s b K-p s b I$ R (5'

\section{AAACTTTGAGAGTAAGCAT 3') or atpF-atpH_R (5'GCTTTTATGGAAGCTTTAACAAT}

3'), with the exception of LemnaMratp152_R, which was amplified using atpF-atpH_F (5'

ACTCGCACACACTCCCTTTCC 3'). Some mismatches were introduced (bold) to increase

PCR specificity for the target species in addition to natural mismatches (underlined). Each

primer name contains the species it amplifies, and the locus and location of the primer-binding site.

\begin{tabular}{|c|c|c|c|c|c|}
\hline Locus & Primer Name & Amplifies & Primer Sequence $\left(5^{\prime} \rightarrow 3^{\prime}\right)$ & $\begin{array}{c}\text { PCR } \\
\text { Product } \\
\text { (bp) }\end{array}$ & $\begin{array}{c}\text { Primer } \\
\text { Annealing } \\
\text { Temperature }\end{array}$ \\
\hline \multirow{4}{*}{$\begin{array}{l}\frac{1}{2} \\
\frac{1}{1} \\
\frac{1}{2}\end{array}$} & LemnaMrpsb97_F & L. minor & GATTCTAAATAAAAATTCATAAGAA息 & 437 & $47^{\circ} \mathrm{C}$ \\
\hline & LemnaTpsb208_F & L. turionifera & TCCATTTCGCCATACA & 326 & $48^{\circ} \mathrm{C}$ \\
\hline & LemnaMapsb178_F & L. minuta & GTATCTTCATTAAAAACAGCAC & 356 & $48^{\circ} \mathrm{C}$ \\
\hline & LemnaGpsb241_F & L. gibba & 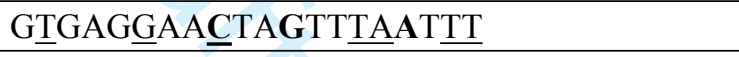 & 293 & $45^{\circ} \mathrm{C}$ \\
\hline \multirow{4}{*}{$\underset{\frac{1}{5}}{\frac{7}{5}}$} & LemnaMratp152_R & L. minor & GGGCGAAGT $\underline{A} A T A G \underline{A A T} \underline{\text { AG }}$ & 523 & $47^{\circ} \mathrm{C}$ \\
\hline & LemnaMaatp236_F & L. minuta & GATTTTTAATGGAATAGAAGGAATTTT & 439 & $49^{\circ} \mathrm{C}$ \\
\hline & LemnaPatp309_F & S. polyrhiza & ATTTATTATTTTAGGCTAATTAAATTAAAGT & 366 & $48^{\circ} \mathrm{C}$ \\
\hline & LemnaGatp394_F & L. gibba & GITACAACGAATACGCT & 281 & $47^{\circ} \mathrm{C}$ \\
\hline
\end{tabular}

* all Lemna GenBank sequences were checked for ATP (L. aequinoctialis, L. disperma, L.

japonica, L. obscura, L. trisulca, and L. valdiviana) and PSB (L. aequinoctialis, L. disperma, L.

japonica, L. obscura, L. tenera, L trisulca, L. valdiviana, and L. yungensis). Primers for L. minor

could amplify L. japonica, L. minuta will amplify L. valdiviana, and L. gibba will amplify $L$.

disperma. 


\section{Figure captions}

Figure 1. Specificity tests. Electrophoresis of DNA extracted from single individuals of $S$. polyrhiza, L. minuta., L. gibba, L. turionifera, L. minor, and L. trisulca using the species-specific primer (listed on far right). Each species has a unique banding profile with sizes of PCR products on the left. A) Banding profiles for atpF-atpH species-specific primers. B) Banding profiles for psbK-psbI species-specific primers.

Figure 2. Sampling locations for Lemna species used in this study. Of the 126 sites, 115 sites contained L. turionifera but not L. minor (red), four contained L. minor but not L. turionifera (yellow) and seven contained both species (black). Full details of sites are in Supplemental Table S1. For map development we used QGIS.org, 2021. QGIS Geographic Information System. (QGIS Association. http://www.qgis.org) 


\section{Figures}

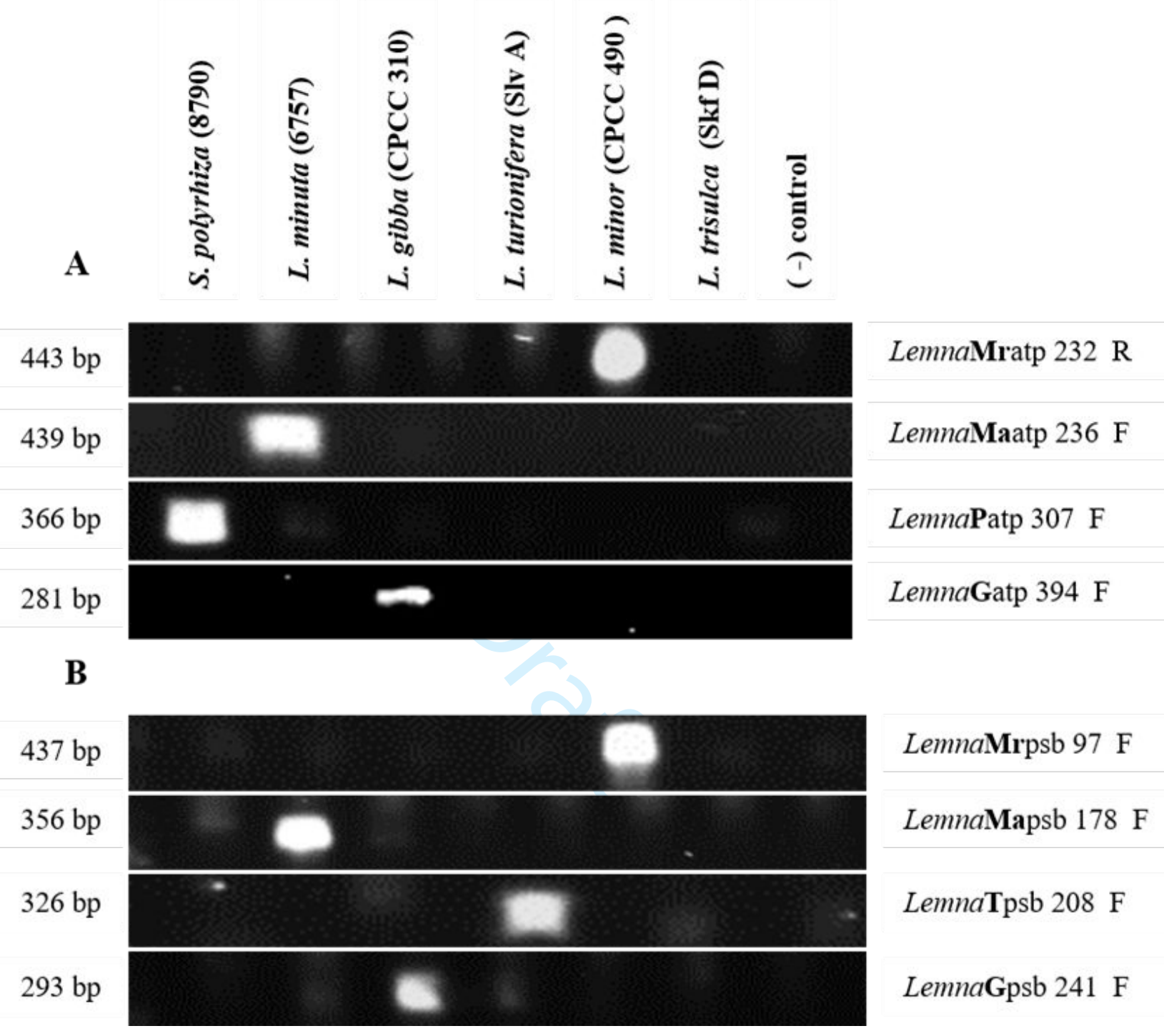

Figure 1 


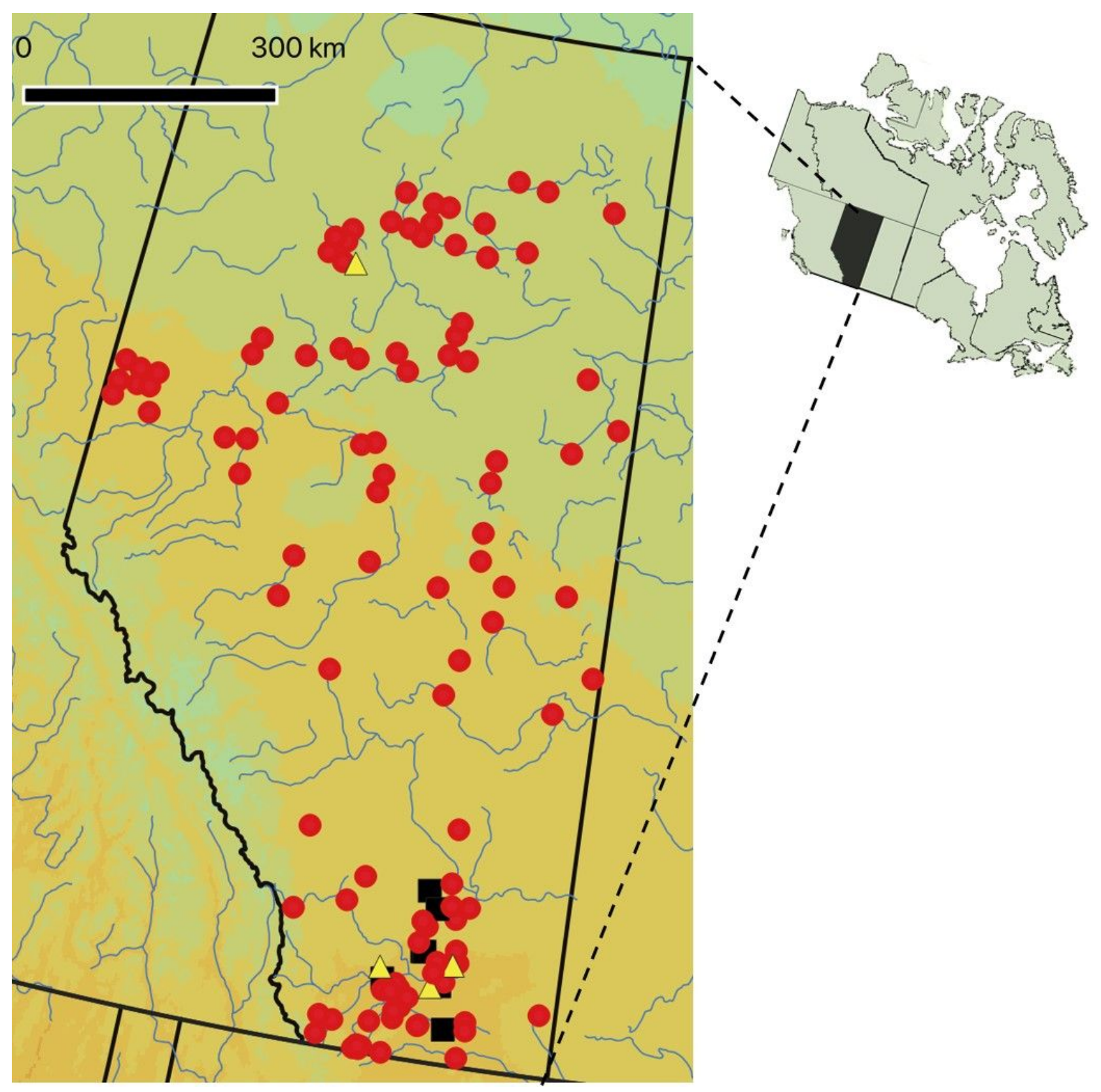

Figure 2 